\title{
Topographic aspects of photic driving in the electroencephalogram of children and adolescents
}

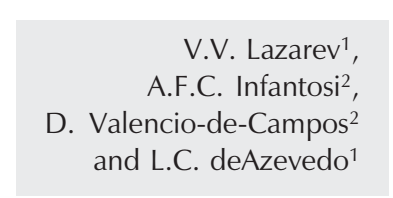

\author{
${ }^{1}$ Laboratório de Neurobiologia e Neurofisiologia Clínica, Setor de Neurologia, \\ Instituto Fernandes Figueira, Fundação Oswaldo Cruz, Rio de Janeiro, RJ, Brasil \\ ${ }^{2}$ Programa de Engenharia Biomédica (COPPE), Centro de Tecnologia, \\ Universidade Federal do Rio de Janeiro, Rio de Janeiro, RJ, Brasil
}

\section{Correspondence \\ V.V. Lazarev \\ Laboratório de Neurobiologia e Neurofisiologia Clínica Instituto Fernandes Figueira FIOCRUZ \\ Av. Rui Barbosa, 716 22250-020 Rio de Janeiro, RJ Brasil \\ Fax: +55-21-2551-0547 \\ E-mail: vlad.v@iname.com}

Received April 27, 2003 Accepted March 22, 2004

\begin{abstract}
The electroencephalogram amplitude spectra at 11 fixed frequencies of intermittent photic stimulation of 3 to $24 \mathrm{~Hz}$ were combined into driving "profiles" for 14 scalp points in 8 male and 7 female normal subjects aged 9 to 17 years. The driving response varied over frequency and was detected in 70 to $100 \%$ of cases in the occipital areas (maximum) and in 27 to $77 \%$ of cases in the frontal areas (minimum) using as a criterion peak amplitude $20 \%$ higher than those of the neighbors. Each subject responded, on average, to $9.7 \pm 1.15$ intermittent photic stimulation frequencies in the right occipital area and to 6.8 \pm 1.97 frequencies in the right frontal area. Most of the driving responses (in relation to the previous background) were significant according to the spectral F-test $(\alpha=0.05)$, which also detected changes in some cases of low amplitude responses not revealed by the peak criterion. The profiles had two maxima in the alpha and theta bands in all leads. The latter was not present in the background spectra in the posterior areas and was less pronounced in the anterior ones. The weight of the profile theta maximum increased towards the frontal areas where the two maxima were similar, while the profile amplitudes decreased. The profiles repeated the shape of the background spectra, except for the theta band. The interhemispheric correlation between profiles was high. The theta driving detected in all areas recorded suggests a generalized influence of the theta generators in prepubertal and pubertal subjects.
\end{abstract}

\section{Introduction}

The driving response to intermittent photic stimulation (IPS) is an important functional test used in electroencephalography (EEG) in order to enhance the manifestation of the latent sources of rhythmic bioelectrical activity including its pathological forms $(1,2)$. In previous communication, we investigated
Key words

- Electroencephalogram

- Intermittent photic

stimulation

- Photic driving

- Brain topography

- Children

- Adolescents 
IPS across the EEG frequency range. These profiles proved to repeat the shape of the background spectra with high Pearson correlation coefficients between them, both having the maximum peaks in the alpha frequency band. However, in the profiles of children and adolescents, the second maximum driving response was detected in the theta band, not present in the background EEG spectra and not described in the literature on the driving reaction in adults. This heightened theta responsiveness was considered to be peculiar for the age group studied. In comparison with the spectrum of the resting EEG, the driving profile suggested the possibility to provide a fuller representation of the set of potential oscillators.

The aforementioned results were described for the right occipital region (3) generally considered representative for the study of the EEG effects of visual stimulation (46 ). However, there is much supporting evidence in the literature for the thesis that the driving response may be observed in other cortical regions as well. Furthermore, the regional specificity may be very important in clinical and psychophysiological diagnosis, especially with respect to local alterations in the functional state of the brain.

First of all, this concerns hemispheric specificity. Various types of interhemispheric differences in the EEG driving reaction were observed in adult subjects and patients (7-9) and it was shown that IPS may emphasize the functional asymmetry (10). It has even been proposed that in certain cases the sensitivity of the left and right hemispheres to IPS may differ in relation to high and low stimulation frequencies (11).

Clinical research has shown that the interhemispheric relationships between the harmonic components of the responses to IPS might be of importance in topographic diagnosis $(12,13)$. The asymmetric driving effects such as a locally increased rhythmic response at the delta or beta frequencies as well as a reduced or a disproportionately strong response within the alpha-band may indicate a focal disturbance and should be taken into account as indicators of a functional alteration (14). Cortical lesions of a destructive type may cause ipsilateral depression or attenuation of driving, whereas irritative lesions, such as those of epileptic scars, may lead to an increased response on the side of the focus $(15,16)$. In paranoid schizophrenia, an increased interhemispheric (17) and decreased intrahemispheric coherence (18) was observed during IPS, revealing, according to the authors, a less lateralized cerebral organization than in normal subjects and a more diffuse, undifferentiated functional organization within the hemispheres. Asymmetrical effects of driving reactions have also been utilized in research on the pathology of the visual field (1).

The regional specificity of the driving reactions in the sagittal direction has been less explored. However, in normal adult subjects, the driving response in the premotor, motor and sensorimotor cortical areas may interfere directly with motor activity (19). The topography of the driving responses at the alpha frequencies permitted the identification and localization of the sources of the alpha rhythm in the thalamic structures by constructing equivalent dipole models (20).

Certain alterations in the sagittal distribution of the driving reaction have been observed in the alpha band in schizophrenic patients. Their lower photic driving occurred across most brain areas except for the centrotemporal regions (21), and in the frontal areas, it could be higher than in the occipital ones (22). Some reduction in photic driving and significant topographic alterations were found in the parieto-occipital regions of patients with presenile Alzheimer's disease (23). In the positron emission tomography of regional blood flow, EEG photic driving correlated with activation of occipital cortical and some subcortical structures, and in photosensitive epileptic patients, the nuclei activated during paroxysmal responses were 
different from those activated in non-paroxysmal driving. For this reason, the latter were associated with an inhibition of epileptic discharges (24).

Thus, the topographic aspect of photic driving looks promising for the study of the regional specificity of the functional state of the brain and its pathological alterations. Some approaches to quantitative EEG topographic mapping may be used in the study of the complex spatial patterns of this phenomenon (25). However, all the aforementioned data concern adult subjects. In our preliminary examination of several children and adolescents suffering from migraine (26), we detected a low spatial consistency in peak frequencies and a decreased interhemispheric correlation of the photic driving profiles by the methodology detailed in the previous communication (3). Thus, the study of the photic driving topography in normal subjects of this age group is very important as a normative basis for clinical studies. However, the weaker response with the lower amplitude in the regions anterior to the occipital visual areas is usually one of the reasons for the latter to be an almost exclusive point of the examination of the driving responses (4-6).

The objective of the present study was to indicate with computerized criteria the presence of the photic driving responses in various brain areas, to assess the sensitivity and physiological significance of these indicators and to compare the driving profiles in different regions in order to reveal the normal topography of the potential latent oscillators not present in the resting EEG and characteristic for the age group studied.

\section{Material and Methods}

The topography of the driving responses was investigated in the same 8 male and 7 female volunteers, between 9 and 17 years of age with a median age of 13.3 , who were described in the previous communication
(3). None was overtly left-handed (according to the writing hand and the reports of the parents) and none had a history of neurological, psychiatric or drug-related illness. The local Ethics Committee approved this research and the subjects or persons responsible gave informed consent to participate in the study.

EEG signals were recorded during a state of relaxed wakefulness (initial background, 2-3-min duration), during IPS of various frequencies and during wakefulness between stimulations at different frequencies. The eyes of the subjects were closed throughout the experiment. Each presentation of a fixed frequency lasted 20-30 s, with the same periods between stimulations. One series of stimulations consisted of the following flicker frequencies: $3,4,5,6,8,10,12,15,18,21$, and $24 \mathrm{~Hz}$.

The EEG was recorded with an 18-channel Nihon Kohden polygraph (EEG-4418), at 14 scalp points, according to the International 10/20 System, with unilateral references to the corresponding earlobes. These points were located over the following areas of the left (odd index) and right (even index) hemispheres: occipital $(\mathrm{O} 1, \mathrm{O} 2)$, parietal ( $\mathrm{P} 3$, $\mathrm{P} 4)$, central $(\mathrm{C} 3, \mathrm{C} 4)$, frontal (F3, F4), posterior temporal (T5, T6), mid-temporal (T3, T4), and anterior temporal (F7, F8). The recording characteristics were: 0.3 -s time constant, $70-\mathrm{Hz}$ high frequency filter and $15-\mu \mathrm{V} / \mathrm{mm}$ sensitivity. The EEG signals were recorded on magnetic tape with a TEAC XR-7000 tape recorder (Tokyo, Japan) with simultaneous paper recording, after digitalization with an $\mathrm{A} / \mathrm{D}$ converter (12-bit precision) at a sampling frequency of $256 \mathrm{~Hz}$. The instants of stimulation were also acquired to be used as reference when applying the signal processing techniques. In order to avoid the possible short post-stimulation effect (27), the first $5 \mathrm{~s}$ after each stimulation were excluded from analysis.

For each IPS frequency, before and during stimulation, the EEG signals were sec- 

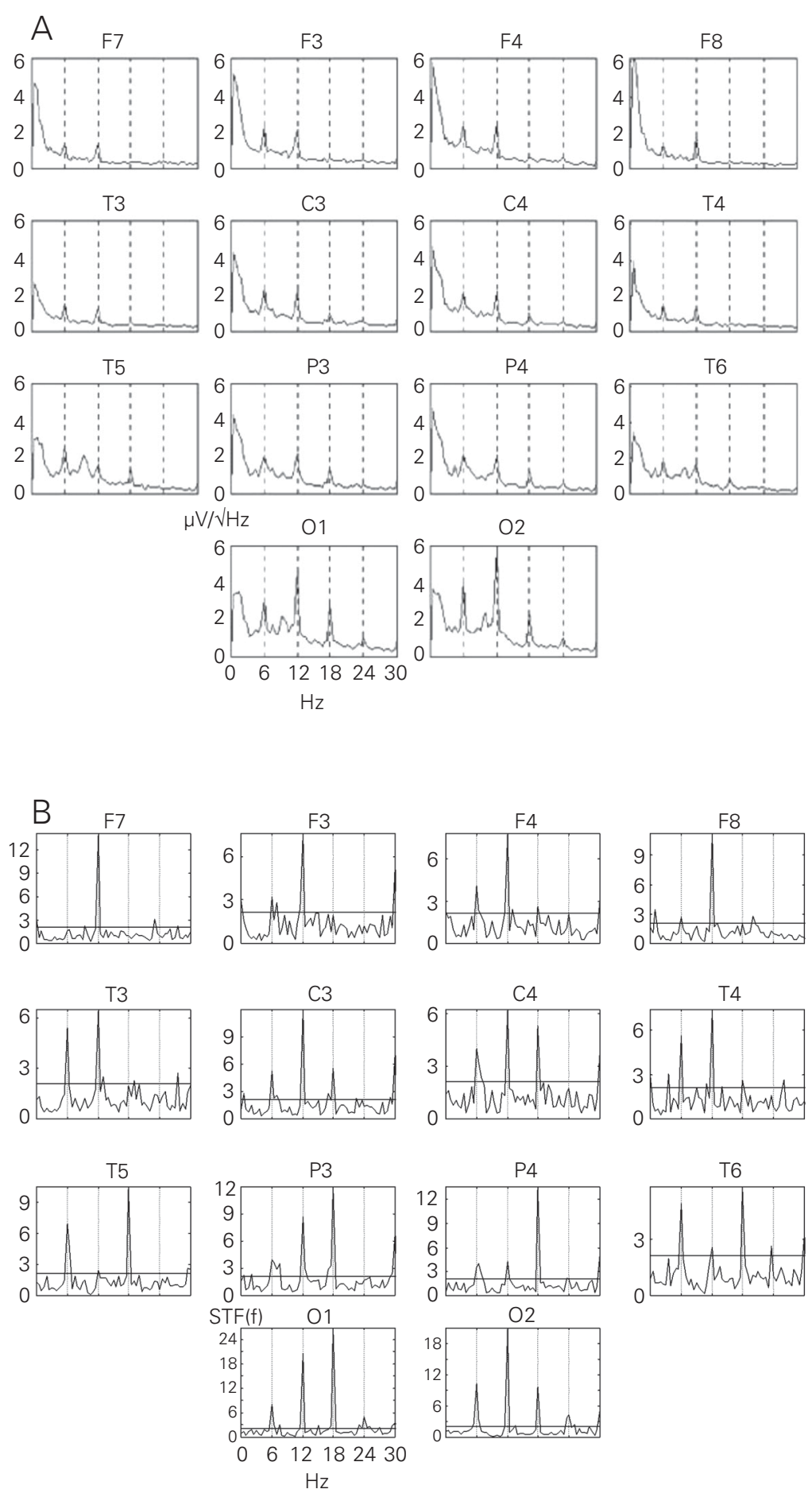

Figure 1. An example of individual spectrograms (A) and spectral F-test value diagrams (B) (subject $\mathrm{M}$ ) with a strong generalized driving response to $6-\mathrm{Hz}$ intermittent photic stimulation in almost all the scalp points recorded (marked above each spectrum or diagram). Abscissas: frequency of EEG, in Hz. Vertical lines mark a frequency of stimulation and its harmonics, i.e., 6, 12, $18 \mathrm{~Hz}$, etc., from left to right. Ordinates: $\mathrm{A}=$ amplitude spectra, in $\mu \mathrm{V} /$ $\sqrt{ } \mathrm{Hz} ; \mathrm{B}=$ spectral F-test values; horizontal line = critical value 2.12 for a level of significance $\alpha=5 \%$. tioned into $\mathrm{M}=10$ epochs of equal duration $(2 \mathrm{~s})$. Then, the spectra were estimated and the spectral F-test (SFT) was applied. The power spectrum was estimated using the modified periodogram of Bartlett based on the Discrete Fourier Transform of the 2-s duration epochs, resulting in a frequency resolution of $0.5 \mathrm{~Hz}$. For each lead, the spectrum was represented as an amplitude spectrum (i.e., the square root of the absolute power) and the presence of the driving response at the frequency of stimulation was ascertained by in-house software as an amplitude peak which was at least $20 \%$ higher than the amplitudes at adjacent frequencies ( $\pm 1 \mathrm{~Hz}$ ). In computer simulation, this level of prevalence gave a false-positive rate of less than $5 \%$ when the spectrum of signals was white, i.e., when there was no response to the stimuli (3). Henceforth, this will be referred to as "the $20 \%$ criterion".

The SFT was calculated as the ratio of the power spectra obtained from the EEG during and before stimulation (28). Knowing that in the absence of spectral change the ratio of averaged periodograms follows an F-distribution, the null hypothesis $\left(\mathrm{H}_{0}\right)$ of the absence of response can be tested (29). Thus, the critical value was determined as $\mathrm{SFT}_{\text {crit }}$ $=2.12$ for a level of significance of $\alpha=5 \%$ and the SFT at any frequency was compared to this value. If $\mathrm{SFT}(\mathrm{f})<\mathrm{SFT}_{\text {crit }}$, the absence of response in the frequency $f$ was accepted, but if SFT(f) $\geq \mathrm{SFT}_{\text {crit }}, \mathrm{H}_{0}$ was rejected.

The results of the spectral analysis were presented for each subject as 14 spectrograms plotted according to the electrode scalp position, as illustrated in Figure 1A. For each lead, the estimated spectrum is depicted as an amplitude spectrum, i.e., the square root of the absolute power, with a frequency resolution of $0.5 \mathrm{~Hz}$. The SFT values were also topographically plotted in the same way (Figure 1B). The frequency of stimulation and its harmonics were indicated by vertical lines (Figure 1).

The group data of the driving presence 
obtained by the $20 \%$ peak criterion and the $\operatorname{SFT}(\alpha=5 \%)$ at each fundamental frequency of stimulation (first harmonic) in each scalp lead were plotted as percent of subjects who responded to the given frequency of IPS at the given lead for the two different schematic scalp maps according to the electrode positions. The two maps were compared by subtraction of the values of the second map from those of the first one.

In order to compare the magnitude of the responses to all the frequencies of stimulation, amplitude spectra measured at all frequencies were plotted on the same diagram called "the frequency profile of the driving reaction" (or simply, "the profile") (3).

The similarity of different profiles at different scalp points was evaluated by the Pearson correlation coefficient (R). The statistical significance of the differences between profiles at each frequency was calculated by the Wilcoxon test.

\section{Results}

The spectrograms of Figure 1A illustrate an example of a strong driving response to the IPS of $6 \mathrm{~Hz}$ in subject M. The figure shows the existence of peaks at the stimulation frequency and at some of its harmonics (12 and $18 \mathrm{~Hz}$ ). In the occipital region, these peaks are more pronounced compared to those of other regions. One may see that the peak amplitudes decrease towards the frontal areas where the third harmonic of $18 \mathrm{~Hz}$ disappears. Furthermore, it is interesting to note that at $12 \mathrm{~Hz}$ (EEG frequency of the alpha band) the relative contribution was higher than at the stimulation frequency (see discussion of this effect in Ref. 3).

Figure 1B depicts the SFT of the 14 EEG leads for the same subject $M$ at the same IPS of $6 \mathrm{~Hz}$. Based on $\mathrm{SFT}_{\text {crit }}=2.12$ (indicated by the horizontal line), the null hypothesis of the absence of response was rejected ( $\alpha=5 \%$ ) (false-positive rate), particularly for the frequency of stimulation and some of its harmonics. For most of the other frequencies, $\mathrm{H}_{0}$ was accepted, that is, no response could be identified. Thus, one can say that in the central, parietal and occipital regions the presence of responses occurs at 6,12 and 18 Hz. Furthermore, in the occipital regions there was also a response at $24 \mathrm{~Hz}$ while in the other ones no responses were identified at this frequency.

Comparing Figure 1A and B one can see that most of the driving responses recorded in all brain areas were detected by both spectral peak (the $20 \%$ criterion) and SFT. However, there were some cases of detection of significant changes in the EEG by SFT against an absence of any visible peak in the spectrum, such as in F4, F8 and T4 at the frequency of $18 \mathrm{~Hz}$. Sometimes, these cases did not relate to the harmonics of the IPS frequency, such as in T4 at 3.5 and 26 $\mathrm{Hz}$, and could be rejected by an increase of the significance level to 2 or $1 \%$. On the other hand, a spectral peak meeting the $20 \%$ criterion could not be confirmed by SFT, such as in $\mathrm{F} 7$ at $6 \mathrm{~Hz}$.

Using the $20 \%$ criterion for the spectrograms, the response to stimulation was most often found, as expected (4-6), in the occipital areas. For the frequency of stimulation (first harmonic), this occurred, on average, in $85 \%$ of subjects (minimum $70 \%$ at $21 \mathrm{~Hz}$ and maximum $97 \%$ at $4 \mathrm{~Hz}$; Figure $2 \mathrm{~A}$ ). However, in the other 12 regions, the driving response was also observed in more than half the subjects at all IPS frequencies except for the $3-\mathrm{Hz}$ case. On average, it was detected in $65 \%$ of subjects - minimum values of $29 \%$ at $3 \mathrm{~Hz}$ and $53 \%$ at $4 \mathrm{~Hz}$ and a maximum value of $72 \%$ at $15 \mathrm{~Hz}$ (Figure 2A). Even in the frontal areas, where the driving response may be expected to be the lowest (4-6), it occurred, on average, in 61\% of cases. In $\mathrm{F} 4$, for example, one subject responded, on average, to $6.8 \pm 1.97$ (mean \pm SD) frequencies of stimulation or to $62 \%$ of the 11 IPS frequencies presented. Only one subject was not responsive to stimulation in 
any region. Therefore, as in our previous communication (3), most of the group data below refer to 14 subjects, excluding the unresponsive subject. In F4, in almost all cases of stimulation, an increase in amplitude spectra relative to the background was observed at all the EEG frequencies corresponding to those of IPS (Figure 3).

When the mapping of the results of the SFT at all stimulation frequencies for all subjects (Figure 2B) was compared with the $20 \%$ criterion maps (Figure $2 \mathrm{~A}$ ), the responses were found to be significant in most cases of the driving peak in the spectrum at the fundamental stimulation frequency (Fig- ure $2 \mathrm{C}$ ). For the general group the number of the significant changes in the EEG at all fundamental frequencies of stimulation was $88.8 \%$ of the peak responses in the occipital areas and $92.6 \%$ in the remaining areas. However, at frequencies of 3 to $15 \mathrm{~Hz}$, in most of the leads (always in the occipital ones), 1 to 6 subjects responded with a spectral peak at the stimulation frequency not confirmed by the SFT. On the other hand, there were some areas where significant changes in the EEG at the stimulation frequency could be observed in some subjects ( 1 to 5 in a lead) without being related to the driving spectral peak. Such areas prevailed
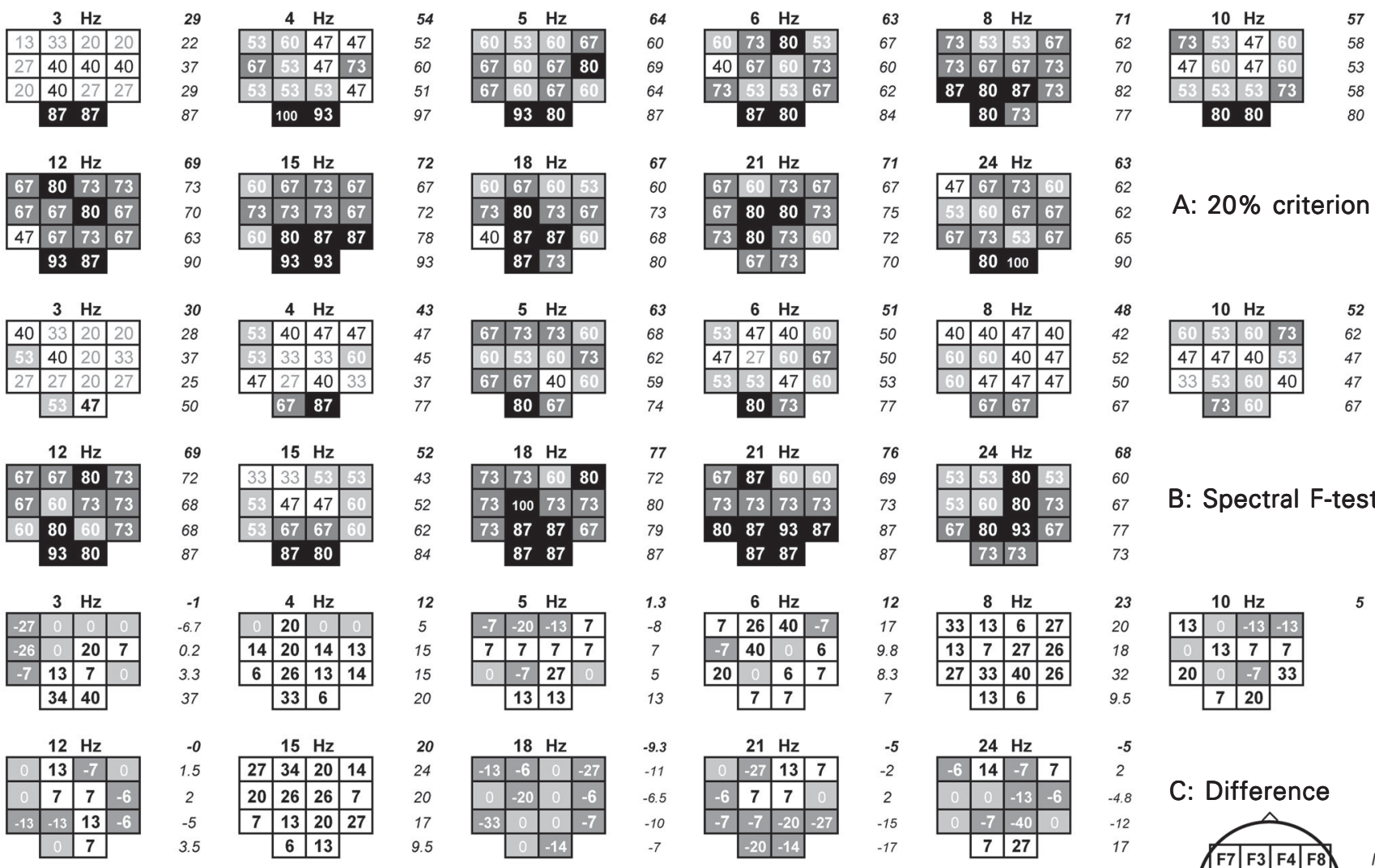

B: Spectral F-test
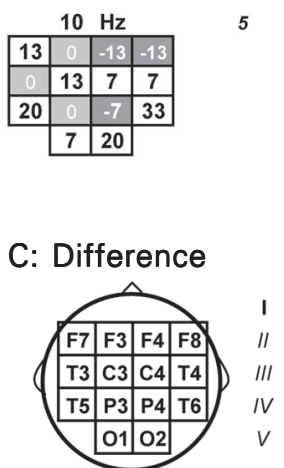

Figure 2. Scalp maps with the percentage of subjects showing a driving response according to the criterion of peak values $20 \%$ greater than those of adjacent frequencies (A) and spectral F-test (B) at each of 11 frequencies of intermittent photic stimulation (IPS, Hz) in each lead. Each square cell represents a lead (plotted on each map according to the head scheme in the right lower corner of the figure) with a percentage for the group of 15 subjects at a frequency of IPS given above the corresponding map. To the right of each map - average data for I: 12 leads except O1 and O2; II, III and IV: 4 leads of a corresponding line; $\mathrm{V}$ : for $\mathrm{O} 1$ and $\mathrm{O} 2$ (see the head scheme in the right lower corner of the figure). C, Maps of difference of the percentages between $20 \%$ criterion and spectral F-test in each lead. 
at frequencies of 18 to $21 \mathrm{~Hz}$. At the lower frequencies and at $24 \mathrm{~Hz}$, this phenomenon was never detected in the occipital regions.

The profiles of the driving responses obtained for the different brain areas proved to have both similar and distinguishing features in comparison with the occipital reactions described in our previous communication (3). For example, this was observed in the right hemisphere where, as mentioned above, the profile in F4 was expected to differ most from that of $\mathrm{O} 2$. The shape of the profiles for $\mathrm{F} 4$ and $\mathrm{O} 2$ (which have different general amplitude levels) can be compared on the basis of the amplitude ratios of the theta, beta and delta maxima to the alpha peak (Figure 3). Both profiles had two maxima in the alpha and theta bands. However, the amplitude ratios between them were different.

In $\mathrm{O} 2$, an increased theta maximum (exceeding half the maximum in the alpha band) was observed in 11 of 14 subjects. It was, on average, $70.5 \pm 14.6 \%$ of the profile's alpha peak amplitude, with the theta maximum never exceeding the alpha one. In the resting EEG, the same ratio was $30.8 \pm 9.9 \%$ (excluding subject $\mathrm{T}$ who did not have an alpha peak in the background EEG). An increase in amplitude spectra at the theta maximum (compared to the background) exceeded half of that in the alpha band in 10 subjects. In these cases, it was, on average, $94.7 \pm 35.4 \%$ of the alpha maximum increase (Figure 3 ). In the remaining 4 subjects, the profile's theta maximum was $38.8 \pm 10.8 \%$ of that of the alpha band, the background ratio was $29.0 \pm$ $11.9 \%$, and the ratio of the maximum amplitude increase in the theta band to that of the alpha band was $39.0 \pm 11.0 \%$.

In $\mathrm{F} 4$, in the same group of 11 subjects who had an increased theta maximum in $\mathrm{O} 2$, such maximum was, on average, $120.7 \pm$ $39.9 \%$ of that of the alpha maximum, exceeding $100 \%$ in 7 cases. In the resting EEG, this ratio was $100.1 \pm 36.7 \%(93.8 \pm 31.9 \%$ without subject $\mathrm{T}$, see above). An increase in amplitude spectra (compared to the background) was at the theta maximum, on average, $179.2 \pm 112.6 \%$ of the alpha maximum increase (Figure 3). In the remaining subjects, the profile's theta maximum was 80.5 $\pm 25.5 \%$ of the alpha one, while the background ratio was $79.7 \pm 28.8 \%$, and the ratio of the maximum theta increase to the alpha increase was $67.3 \pm 15.5 \%$.

The ratio of the profile beta maximum to the profile alpha maximum was also higher in $\mathrm{F} 4$ than in $\mathrm{O} 2$. In F4, in 14 subjects it was $52.6 \pm 17.9 \%$ in the driving profile, $83.2 \pm$ $47.6 \%(>100 \%$ in the same 5 subjects who displayed such prevalence in the theta band)
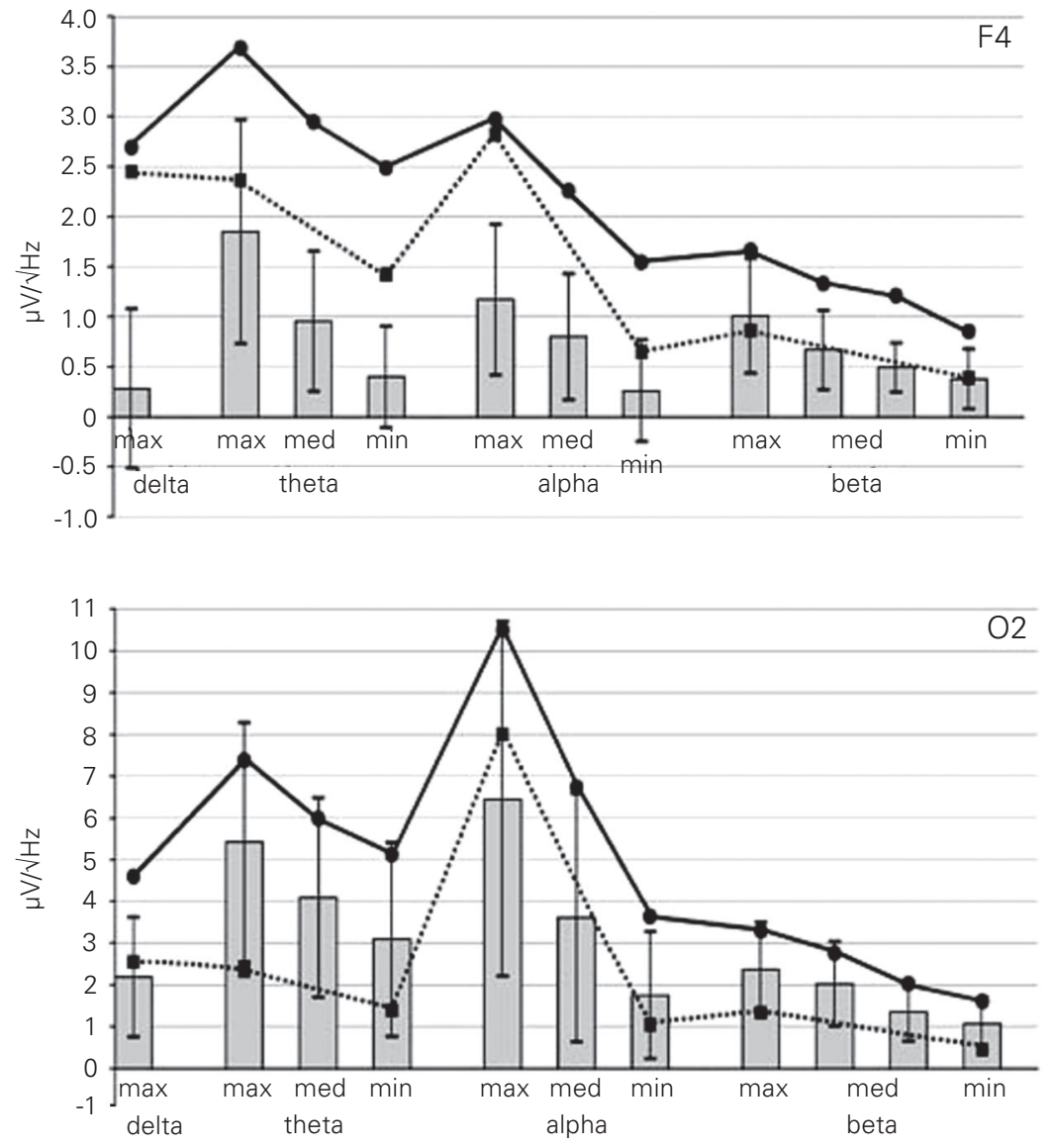

Figure 3. Group average driving profiles in the right frontal (F4) and occipital (O2) areas Ordinate: amplitude spectra, in $\mu \mathrm{V} / \mathrm{JHz}$ individual values for each subject being ranged before averaging for each frequency band from maximum to minimum. Abscissa: frequency of intermittent photic stimulation and of EEG. Solid line: driving profile; dashed line: initial background; bars: change in amplitude spectra during stimulation in relation to the background 
in the amplitude increase in relation to the background, and $36.4 \pm 12.1 \%$ in the background. In $\mathrm{O} 2$ these ratios were $32.5 \pm 15.5 \%$ in the driving profile, $45.1 \pm 33.9 \%$ in the amplitude increase, and $18.6 \pm 6.1 \%$ in the background. Nevertheless, in F4, the profile maximum in the beta band was only $49.2 \pm$ $10.9 \%$ of the alpha peak (Figure 3 ).

In F4, a relatively high amplitude of the driving response to IPS of $3 \mathrm{~Hz}$ was also observed, corresponding to $86.0 \pm 25.7 \%$ of
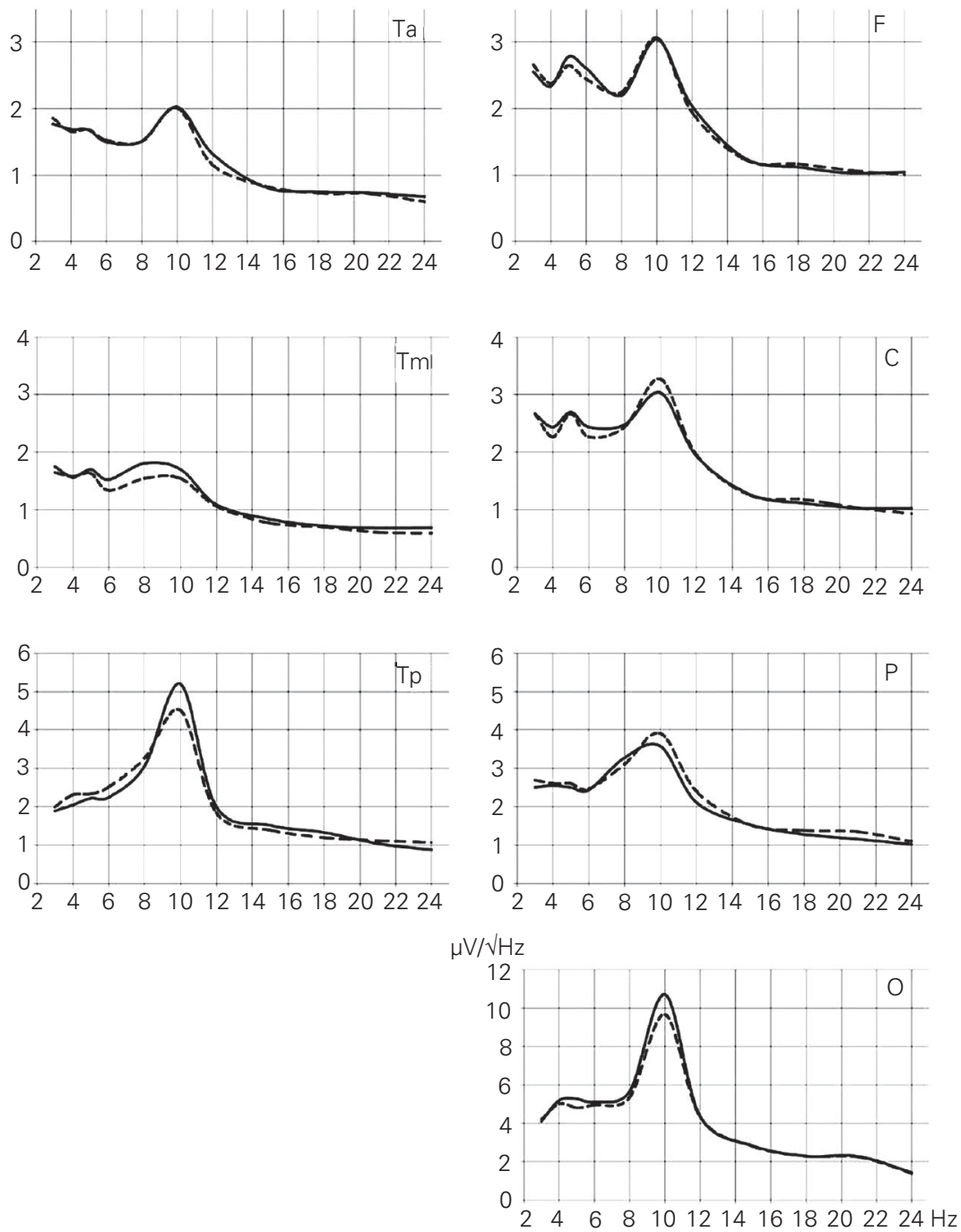

Figure 4. Average driving profiles for 10 subjects with the peak alpha frequency of $10 \mathrm{~Hz}$, in the frontal $(F)$, central $(C)$, parietal $(P)$, occipital $(\mathrm{O})$, anterior temporal $(\mathrm{Ta})$, temporal $(\mathrm{Tm})$, and posterior temporal (Tp) areas. Ordinate: amplitude spectra, in $\mu \mathrm{V} / \mathrm{VHz}$. Abscissa: frequency of intermittent photic stimulation and EEG. Solid line: profile for the right hemisphere; dashed line: profile for the left hemisphere. the alpha peak. This ratio was substantially higher than $42.7 \pm 16.7 \%$ in O2. However, one can see in Figure 3 that a similar interregional ratio occurred in the resting EEG, i.e., $97.9 \pm 26.7 \%$ and $36.5 \pm 18.1 \%$, respectively.

The specificity of the ratios between the amplitudes of the driving reaction in different frequency bands appears to depend on the frequency structure of the background EEG. In F4, similarly to O2 (3), a high level of correlation between profiles and background spectra was observed, with an average correlation coefficient $\mathrm{R}_{\mathrm{db}}=0.75 \pm 0.15$ $\left(\mathrm{P}<0.001\right.$; in $\left.\mathrm{O} 2, \mathrm{R}_{\mathrm{db}}=0.82 \pm 0.13\right)$. However, in $\mathrm{O} 2$, a decreased value of such a correlation in some subjects was caused predominantly by the appearance of an additional maximum in the theta band (not present in the background spectrum) and its relative weight in the overall profile. The ratio of the amplitude spectra of this maximum to that of the alpha peak was negatively correlated with the values of $R_{d b}(R=-0.77, P<$ 0.001) (3). In F4, such a decrease in $R_{d b}$ could depend on a disproportionately increased driving response not only in the theta band but also in the beta band. This was reflected on the negative correlation of the values of $R_{d b}$ with the amplitude spectra at the maxima in both the theta $(\mathrm{R}=-0.78, \mathrm{P}<$ $0.001)$ and beta $(\mathrm{R}=-0.69, \mathrm{P}<0.01)$ bands.

The most pronounced theta driving in the frontal and occipital areas was observed in the same subjects: the theta maxima in these leads correlated in this group with $\mathrm{R}=0.75$ $(\mathrm{P}<0.001)$.

In the brain areas of the same hemisphere situated between the frontal and occipital poles, an intermediate characteristic of the driving profile form was observed. This was reflected on the lower values of the Pearson correlation coefficient between the profiles of relatively more distant scalp points (Figure 4). The driving profile for $\mathrm{O} 2$ correlated with that of $\mathrm{P} 4(\mathrm{R}=0.80 \pm 0.12, \mathrm{P}<0.001)$, $\mathrm{C} 4(\mathrm{R}=0.66 \pm 0.23, \mathrm{P}<0.01)$, and $\mathrm{F} 4(\mathrm{R}=$ 
$0.68 \pm 0.21, \mathrm{P}<0.01)$. The profile of $\mathrm{F} 4$ correlated with that of $\mathrm{C} 4(\mathrm{R}=0.94 \pm 0.06$, $\mathrm{P}<0.001)$ and $\mathrm{P} 4(\mathrm{R}=0.74 \pm 0.23, \mathrm{P}<$ $0.001)$.

The topographic differences described here in the profile shape occurred mainly due to a general decrease in the relative weight of the alpha activity towards the frontal region. However, a less pronounced decrease in the voltage of the driving reaction was also observed in the other bands (Figure 4). For example, with respect to the 14 subjects, the average amplitude of the profile alpha peak in F4 was $3.49 \pm 1.44 \mu \mathrm{V} / \sqrt{ } \mathrm{Hz}^{\mathrm{a}}$ or $32.3 \%$ of $10.81 \pm 3.17 \mu \mathrm{V} / \sqrt{ } \mathrm{Hz}$ in $\mathrm{O} 2$, in the right hemisphere. In contrast, the profile maximum in the theta band was $3.61 \pm 1.38$ $\mu \mathrm{V} / \sqrt{\mathrm{Hz}}$ or $54.4 \%$ of $6.64 \pm 2.79 \mu \mathrm{V} / \sqrt{\mathrm{Hz}}$ in $\mathrm{O} 2$. With this exception, a certain difference between $\mathrm{F} 4$ and $\mathrm{O} 2$ was detected in the peak profile frequencies in several cases, thereby contributing to decreasing somewhat the similarity between the profiles. From $\mathrm{O} 2$ (average alpha frequency $9.57 \pm 1.16 \mathrm{~Hz}$ ) to $\mathrm{F} 4$ $(9.43 \pm 1.45 \mathrm{~Hz})$, the frequency of the profile alpha peak decreased in 2 cases and increased in 1 case. In the theta band, there were both an increase and a decrease of the profile peak frequency from $\mathrm{O} 2(4.86 \pm 0.86$ $\mathrm{Hz})$ to $\mathrm{F} 4(4.93 \pm 0.73 \mathrm{~Hz})-5$ cases each.

In the left hemisphere, the profile shapes and the amplitude ratios of the profile maxima in the different bands were very similar since there was a high interhemispheric similarity between the symmetrical scalp point profiles (Figure 4). Relatively lower average Rs were found between the posterior temporal $(\mathrm{R}=$ $0.88 \pm 0.08, \mathrm{P}<0.001)$ and the mid-temporal $(\mathrm{R}=0.86 \pm 0.13, \mathrm{P}<0.001)$ leads due to the lower values in some subjects, whereas in the other area, the average $R$ was $0.94 \pm 0.05$ $(\mathrm{P}<0.001)$.
In the alpha band, the prevalence of the profile peak amplitude in the right hemisphere was significant only in the occipital areas $(\mathrm{P}<0.05)$ where the average ratio of the right peak amplitude to the left one was 1.12 , the right-side prevalence (ratio $>1.0$ ) being observed in 10 of 14 subjects. In most cases ( $88 \%$ ), the profile peak alpha frequency in symmetrical leads was the same.

In the theta band, the significant rightside prevalence of the profile maximum $(\mathrm{P}<$ 0.05 ) was also observed only in the occipital areas, with an average interhemispheric ratio of 1.10 ( $>1.0$ in 9 subjects).

\section{Discussion}

The present results show that in the preadolescent and adolescent children examined the high responsiveness to the IPS was characteristic for all brain regions. Even in the frontal ones, an increase in amplitude spectra relative to the background was observed in the most subjects at all EEG frequencies. Such an increase may serve as an additional driving response indicator if we consider that the non-specific orienting reaction to photic stimuli usually produces an amplitude decrease at the adjacent frequencies $(3,5,30)$. It may be supposed that the driving reactivity in the age group studied is increased in comparison with that of adults not only in the occipital areas, as it was noted in our previous communication (3) on the basis of literature data $(1,2,16)$, but also in the other areas.

The spectral peak criterion for the detection of the driving response proposed in our previous communication (3) proved to be useful in the topographic study of the phenomenon, especially together with the SFT. An application of the latter is very important

aThe unit of the power spectrum is $\mu \mathrm{V}^{2} / \mathrm{Hz}$, as habitually used for the background EEG, and that of the amplitude spectrum therefore is $\mu \mathrm{V} / \mathrm{VHz}$. This will be used throughout the current work. In the driving reaction, consisting of sinusoidal oscillations at a number of well-defined frequencies, the amplitudes of the harmonics (in $\mu \mathrm{V}$ ) can be calculated by multiplying the amplitude spectral values at the corresponding frequencies by 1.41 (for the spectral resolution of $0.5 \mathrm{~Hz}$ employed). 
for a statistical evaluation of the reactions to the IPS, and it did confirm the significance of most of the responses obtained, even of the low-amplitude ones in the anterior regions.

However, the discordant cases of the spectral peak and of statistical criteria are worthy of special consideration. According to the physiological conception of the driving response, the resonance frequency selectivity is its principal feature. Thus, the detection of changes in the EEG at the IPS frequency by the SFT without a spectral peak may be considered to be a driving response only when there are no changes at the adjacent EEG frequencies. Otherwise, the changes, even when related to the IPS but not selective for its narrow frequency, may be nonspecific, such as the EEG desynchronization as part, for example, of the orienting reaction and synchronization in the habituation $(5,30)$. This type of EEG desynchronization/ synchronization, in spite of being most pronounced in the alpha band, may take place in all other bands $(31,32)$.

In some cases of selective EEG changes detected by the SFT only at the IPS frequency, SFT proved to be a more sensitive driving indicator than the spectral peak criterion, at least when the latter was taken at the $20 \%$ level of excess over the adjacent frequencies. Indeed, most cases of SFT indications not accompanied by the spectral peak criterion fall on the anterior areas and on the higher beta frequencies when the driving responses usually are of low amplitude. This needs further investigation.

On the other hand, the orienting reaction during IPS mentioned above may also result in the spectral peak not confirmed by the SFT. In such a case, its effect of non-specific general EEG desynchronization neutralizes the selective driving synchronization, and the latter forms a driving spectral peak without significant EEG changes at the stimulation frequency (or its harmonics) due to an amplitude decrease at the adjacent frequen- cies. We described such a case for the alpha band in the previous communication (3). This may partially explain a relative prevalence of these cases in the occipital areas at the low frequencies of IPS presented at the beginning of stimulation series when the orienting reaction must be most pronounced.

Thus, the two criteria used for the driving response detection proved to be quite reliable, sensitive and mutually complementary instruments in reviewing the estimate of the driving topography.

The construction of an individual driving profile proved to be a quite convenient and informative tool for a detailed assessment of the driving responses and for the quantitative comparison of the reactions to different frequencies of IPS in different regions. The profiles for the photic driving induced by stimulation frequencies from 3 to $24 \mathrm{~Hz}$ showed that all the main features described for the right occipital area (3) were valid for all the other regions. Their correlation with the background EEG spectra, showing maximum responses at the dominating frequencies alpha and theta, further supports the resonance nature of the driving reaction $(4,21,27,33-35)$ discussed in our previous work (3). This correlation also means that in all brain regions, the reactivity to a given IPS frequency is relatively stable since the magnitude of the response is usually proportional to the background amplitude spectra at the same EEG frequency, regardless of the stimulation times which are different for different IPS frequencies. This makes profiles maintain their shape in time and principal amplitude proportions between the frequency bands characteristic for the background spectrum but on a higher amplitude level. Thus, the profile may be treated as somewhat equivalent to a spectrum (3).

On the other hand, profiles may reflect the latent power of some neuronal oscillators $(1,36-39)$ such as those of the theta peaks revealed in children and adolescents in our previous (3) and present research. In 
the background spectra, these peaks were not observed in the occipital areas and were less expressed in the other ones. This permits us to consider the driving profile to be a more complete representation of an individual's set of potential oscillators in comparison with the resting EEG spectrum. In the profile, the manifestation of these oscillators may be seen at a higher synchronization level, presumably closer to the maximum in certain cases, such as those of the alpha band considered in our previous communication (3). For this reason, regarding the complex characteristics of an individual's EEG, sometimes it practically seems less important whether such a maximum in a profile is reached due to the resonance-like driving mechanisms or beyond them (spontaneously), although the magnitude of the driving response itself in relation to the background level reflects some special individual neurophysiological mechanisms of certain importance for psychophysiological and clinical research and diagnostics. The problem is that this magnitude is dependent on the background state which is difficult to control. The profile must reflect a relatively more standardized functional state of the brain due to external stimulation considered to minimize uncontrolled subjective variations in a current state (39). This demonstrates another advantage of the profile, which was found to be more stable than a resting EEG spectrum in an individual (3). The longterm stability of the frequencies of the individual driving reactivity in adults has been considered to reflect constitutional features of the EEG $(33,35,40)$.

Such individual stability, together with features differing from those of adults, enables us to propose the use of the driving profile in children and adolescents as an indicator of the ontogenesis of the electrical activity of the brain and as an individual characteristic of a developmental stage. The principal peculiarity of the profile during the prepubertal and pubertal periods seemed to be the additional theta peak, which was not present in the resting EEG spectrum of the occipital areas (3). The study of the driving reaction topography demonstrates an increase of relative weight of the theta peak in the profile up to its predominance in the anterior areas. Even in the frontal region, where the theta peak may be seen in the resting EEG spectra, the IPS selectively augments its weight in comparison with the profile alpha peak. The results show that, in preadolescents and adolescents, the influence of the mechanisms of generation of theta activity is topographically more generalized than it seems to be in the resting EEG. This may probably reflect the resting EEG structure in the previous stages of development for which an increased spectral power of theta and delta activities is characteristic (2). This needs further investigation in younger age groups.

The responsiveness to at least $2 / 3$ of the IPS frequencies presented, revealed in almost any cortical area, proves to be of diagnostic value for the topographic aspect of the driving reaction in children and adolescents. This is a very promising development for functional brain mapping and its clinical applications. Therefore, the normative data about the peculiarities of the profile shape in various cortical areas of healthy children and adolescents are important as controls for clinical studies. The topographic parameters of the normal driving reaction, such as the interhemispheric symmetry of the profiles with some prevalence of the right hemisphere in the occipital areas, their gradual amplitude decrease in the frontal direction with a decrease of intrahemispheric correlation and an increase of the weight of the theta peak, etc., can be compared with those of the patients with regional alterations in cerebral functioning of both an organic and functional nature (14-18,21-23). Our preliminary results obtained for some children suffering from migraine actually demonstrated a pronounced interhemispheric asymmetry in the profile shape not observed in the resting 
EEG spectra. They may probably reflect some deficiency in the interhemispheric coordination of the functioning of neuronal oscillators of different frequencies (26). This finding seems promising for the study of the neurophysiological mechanisms of this type of pathology, and may be important in elaborating electrophysiological methodologies for diagnostic purposes. The data confirm the position of some authors that the features of the driving reactions to IPS constitute the most reliable EEG indicators for the different types of headaches $(37,38)$, and show that the topographic aspect may substantially increase the informative value of this methodology. Exploring the topographic characteristics of the driving responses may be very important in EEG studies and for the neurophysiological attributions of regional alterations in the functional state of the brain.

\section{Acknowledgments}

The authors are grateful to Dr. D.M. Simpson for his help with part of the software and for useful discussion.

\section{References}

1. Takahashi T (1993). Activation methods. In: Niedermeyer E (Editor), Electroencephalography: Basic Principles, Clinical Applications, and Related Fields. Williams \& Wilkins, Baltimore, MD, USA.

2. Kooi KA, Tucker RP \& Marshall RE (1978). Fundamentals of Electroencephalography. Harper \& Row, Hagerstown, MD, USA.

3. Lazarev VV, Simpson DM, Schubsky BM \& deAzevedo LC (2001). Photic driving in the electroencephalogram of children and adolescents: harmonic structure and relation to the resting state. Brazilian Journal of Medical and Biological Research, 34: 1573-1584.

4. Walter VJ \& Walter WG (1949). The central effects of rhythmic sensory stimulation. Electroencephalography and Clinical Neurophysiology, 1: 57-86.

5. Yaguchi K \& Iwahara S (1976). Temporal sequence of frequency specific and nonspecific effects of flickering lights upon the occipital electrical activity in man. Brain Research, 107: 27-38.

6. Fox PT \& Raichle ME (1984). Stimulus rate dependence of regional cerebral blood flow in human striate cortex, demonstrated by positron emission tomography. Journal of Neurophysiology, 51: 1109-1120.

7. Freedman NL (1963). Bilateral differences in the human occipital EEG with unilateral photic driving. Science, 142: 598-599.

8. Lansing RW \& Thomas H (1964). The laterality of photic driving in normal adults. Electroencephalography and Clinical Neurophysiology, 16: 290-294

9. Binnie CD, Wilkins AJ \& de Korte RA (1981). Interhemispheric differences in photosensitive epilepsy. II. Intermittent photic stimulation. Electroencephalography and Clinical Neurophysiology, 52: 469-472.

10. Hirota T, Yagyu T, Pascual-Marqui RD, Saito N \& Kinoshita T (2001). Spatial structure of brain electric fields during intermittent photic stimulation. Neuropsychobiology, 44: 108-112.

11. Fedotchev Al \& Bondar AT (1990). Human EEG reactions to the intermittent photic stimulation of different frequency. Uspekhi Fiziologicheskikh Nauk, 21: 97-109 (in Russian).

12. Donker DN (1975). Harmonic composition and topographic distribution of responses to sine wave modulated light (SML), their reproducibility and their interhemispheric relationship. Electroencephalography and Clinical Neurophysiology, 39: 561-574.

13. Donker DN, Njio L, van Leeuwen WS \& Wieneke G (1978). Inter- hemispheric relationships of responses to sine wave modulated light in normal subjects and patients. Electroencephalography and Clinical Neurophysiology, 44: 479-489.

14. Scheuler W (1983). Clinical significance of increased reaction to photostimulation in the alpha frequency range. EEG-EMG Zeitschrift für Elektroenzephalographie, Elektromyographie und Verwandte Gebiete, 14: 143-153 (in German).

15. Bickford RG (1979). Activation procedures and special electrodes. In: Klass DW \& Daly DD (Editors), Current Practice of Clinical Electroencephalography. Raven Press, New York.

16. Beydoun A, Schechter SH, Nasreddine W \& Drury I (1998). Responses to photic stimulation in patients with occipital spikes. Electroencephalography and Clinical Neurophysiology, 107: 13-17.

17. Wada Y, Nanbu Y, Jiang ZY, Koshino Y \& Hashimoto T (1998). Interhemispheric EEG coherence in never-medicated patients with paranoid schizophrenia: analysis at rest and during photic stimulation. Clinical Electroencephalography, 29: 170-176.

18. Wada Y, Nanbu Y, Kikuchi M, Koshino Y \& Hashimoto T (1998), Aberrant functional organization in schizophrenia: analysis of EEG coherence during rest and photic stimulation in drug-naive patients. Neuropsychobiology, 38: 63-69.

19. Petrenko ET (1988). Electroencephalographic changes in response to the equilibrium test and rhythmic light flashes. Kosmicheskaya Biologiya i Aviakosmicheskaya Meditsina, 22: 21-25 (in Russian).

20. Isaichev SA, Dereviankin VT, Koptelov luM \& Sokolov EN (1999). Generators of the rhythmic alpha activity in the human EEG. Zhurnal Vysshei Nervnoi Deiatelnosti Imeni I.P. Pavlova, 49: 919-925 (in Russian).

21. Jin Y, Castellanos A, Solis ER \& Potkin SG (2000). EEG resonant responses in schizophrenia: a photic driving study with improved harmonic resolution. Schizophrenia Research, 44: 213-220.

22. Wada $Y$, Takizawa $Y$ \& Yamaguchi $N$ (1995). Abnormal photic driving responses in never-medicated schizophrenia patients. Schizophrenia Bulletin, 21: 111-115.

23. Nanbu $Y$, Wada $Y$, Nakajima $M$, Futamata $H$, Hashimoto $T$ \& Koshino Y (1997). Topographic analysis of resting EEG and photic driving responses in patients with presenile Alzheimer's disease. Rinsho Byori, 45: 277-281 (in Japanese).

24. da Silva EA, Muller RA, Chugani DC, Shah J, Shah A, Watson C \& 
Chugani HT (1999). Brain activation during intermittent photic stimulation: a [150]-water PET study on photosensitive epilepsy. Epilepsia, 40 (Suppl 4): 4-17-4-22.

25. Rau R, Raschka C \& Koch HJ (2002). Uniform decrease of alphaglobal field power induced by intermittent photic stimulation of healthy subjects. Brazilian Journal of Medical and Biological Research, 35: 605-611.

26. Lazarev V, Simpson DM, Schubsky BM \& deAzevedo LC (2000). Harmonic structure and topography of EEG driving reaction to intermittent photic stimulation in children and adolescents. In: Maurer K \& Dierks $T$ (Editors), The Eleventh World Congress. International Society for Brain Electromagnetic Topography. ISBET 2000 Millenium. Abstract Book. Frankfurt, Germany, November 16-19, 2000 (Abstract), 50.

27. Sakamoto H, Inouye T \& Shinosaki K (1993). Preservation of alpha rhythm shortly after photic driving. International Journal of Neuroscience, 73: 227-233.

28. Tierra-Criollo CJ, Simpson DM \& Infantosi AFC (1998). Detección objetiva de respuestas evocadas en el EEG con la prueba espectral F ponderada [Objective Detection of Evoked Responses in the EEG with the Weighted Spectral F Test]. In: I Congresso LatinoAmericano de Ingeniería Biomédica. Vol. 1. Mazatlan, Sinaloa, Mexico, November 11-14, 1998.

29. Infantosi AFC, Lazarev W \& Valencio-de-Campos D (2002). O teste F-espectral na detecção de resposta no EEG durante fotoestimulação intermitente. In: XVIII Congresso Brasileiro de Engenharia Biomédica, São José dos Campos, SP, Brazil, September 9-12, 2002.

30. Morrell LK (1966). Some characteristics of stimulus provoked alpha activity. Electroencephalography and Clinical Neurophysiology, 21: 552-561.

31. Petsche $H$, Rappelsberger $P$ \& Rockberger $H$ (1988). Sex differences in ongoing EEG: Probability mapping at rest and during cognitive tasks. In: Pfurtscheller G \& Lopes da Silva FH (Editors), Func- tional Brain Imaging. Hans Huber, Toronto, Canada.

32. Lazarev V (1998). On the intercorrelation of some frequency and amplitude parameters of the human EEG and its functional significance. Communication I. Multidimensional neurodynamic organization of functional states of the brain during intellectual, perceptive and motor activity in normal subjects. International Journal of Psychophysiology, 28: 77-98.

33. Fedotchev Al, Bondar AT \& Konovalov VF (1990). Stability of resonance EEG reactions to flickering light in humans. International Journal of Psychophysiology, 9: 189-193.

34. Basar E, Basar-Eroglu C, Rahn E \& Schurmann M (1991). Sensory and cognitive components of brain resonance responses. Acta OtoLaryngologica (Suppl 491): 25-34.

35. Salansky N, Fedotchev A \& Bondar A (1998). Responses of the nervous system to low frequency stimulation and EEG rhythms: clinical implications. Neuroscience and Biobehavioral Reviews, 22: 395-409.

36. Drake Jr ME, Shy KE \& Liss L (1989). Quantitation of photic driving in dementia with normal EEG. Clinical Electroencephalography, 20: 153-155.

37. Genco S, de Tommaso M, Prudenzano AM, Savarese M \& Puca FM (1994). EEG features in juvenile migraine: topographic analysis of spontaneous and visual evoked brain electrical activity: a comparison with adult migraine. Cephalalgia, 14: 41-46.

38. Gronseth GS \& Greenberg MK (1995). The utility of the electroencephalogram in the evaluation of patients presenting with headache: a review of the literature. Neurology, 45: 1263-1267.

39. Kaiser J \& Gruzelier JH (1996). Timing of puberty and EEG coherence during photic stimulation. International Journal of Psychophysiology, 21: 135-149.

40. Tyler CW, Apkarian P \& Nakayama K (1978). Multiple spatial-frequency tuning of electrical responses from human visual cortex. Experimental Brain Research, 33: 535-550. 